\title{
12. Massimis milder kantischer Realismus
}

Von Michela Massimi wird in jüngster Zeit eine in der zeitgenössischen Realismusdebatte bisher unbeachtete Theorieoption ins Spiel gebracht. Massimi plädiert für ein kantisches Phänomenverständnis und eine damit verbundene milde kantische Version des Realismus. Diesen Vorschlag möchte ich in diesem Kapitel genauer untersuchen, da auch für Massimi die Daten-Phänomen-Unterscheidung den Ausgangspunkt ihrer Überlegungen darstellt.

Massimi diagnostiziert, dass die zeitgenössische Realismusdebatte in einer Sackgasse stecke: Der Wissenschaftliche Realismus sei in jüngster Zeit insbesondere durch Angriffe bedrängt worden, die ihn an seiner epistemologischen Flanke attackieren. Argumente wie die Unterbestimmtheitsthese oder die pessimistische Metainduktion schüren Zweifel an der realistischen Behauptung, dass unsere aktuell besten Theorien tatsächlich Wissen über uns nicht in der Wahrnehmung zugängliche Bereiche der Wirklichkeit vermitteln. ${ }^{300}$ Insbesondere der Konstruktive Empirismus stoße in diese Lücke und biete eine Alternative zum Realismus an, die epistemisch bescheidener sei und sich deshalb den Schwierigkeiten, die die genannten Argumente hervorrufen, nicht aussetze. Gleichzeitig aber rufe der Konstruktive Empirismus aufgrund der Diskrepanz zwischen denjenigen Phänomenen, die es van Fraassen zufolge zu retten gilt (das sind, wie wir in den Kapiteln 2 und 9 sahen, beobachtbare Sachverhalte), und denjenigen Phänomenen, mit denen sich eine Vielzahl von Wissenschaftlern in ihrer täglichen Praxis auseinandersetzen, nämlich dem (vermeintlichen) Verhalten unbeobachtbarer Entitäten wie Viren, Bakterien, Atome, Gene, Quarks usw., eine nicht unerhebliche Irritation hervor. ${ }^{301}$ Einer von Bogens und Woodwards Verdiensten sei es, auf dieses Missverhältnis hingewiesen zu haben. Ihre Daten-Phänomen-Unterscheidung liege quer zur van Fraassen'schen Beobachtbar-unbeobachtbar-Unterscheidung und lasse

\footnotetext{
${ }^{300}$ Vgl. Massimi (2008), S. 2.

${ }^{301}$ Eine detaillierte Ausarbeitung von Massimis Kritik an van Fraassen findet sich in Massimi (2007). Da ich in Kapitel 9 bereits ausgearbeitet habe, dass es Bogens und Woodwards Argumentation über die Daten-Phänomen-Unterscheidung nicht erlaubt, den Konstruktiven Empirismus zurückzuweisen, und sich auch Massimis Kritik an van Fraassen wesentlich auf diese stützt, gehe ich auf Massimis Einwände gegen den Konstruktiven Empirismus nicht weiter ein.
} 
deshalb den Konstruktiven Empirismus wenig plausibel erscheinen. ${ }^{302}$ Massimi konstatiert letztlich ein Patt zwischen Wissenschaftlichen Realisten und Antirealisten. ${ }^{303}$ Diese Pattsituation möchte sie durch die Einführung einer neuen, bisher in der Debatte unberücksichtigten Theorieoption überwinden. ${ }^{304}$ Sie schlägt eine kantische Konzeption naturwissenschaftlicher Phänomene vor, die weder eine Form des Wissenschaftlichen Realismus noch eine Variante gängiger Antirealismen sein soll. Ihrer Auffassung zufolge entdecken Naturwissenschaftler keine von uns unabhängigen Phänomene, sondern wissenschaftliche Phänomene seien begrifflich verfasste Erscheinungen („conceptualised appearances“) in einem kantischen Sinne, die in mindestens einer wesentlichen Hinsicht durch uns konstituiert würden. ${ }^{305}$ Nichtsdestotrotz soll Massimis Position keine Spielart eines konstruktivistischen oder konventionalistischen Antirealismus sein, sondern zentrale Aspekte der realistischen Auffassung bewahren. In diesem Kapitel soll der Frage nachgegangen werden, was Massimis kantische Phänomenkonzeption genau besagt, und ob sie tatsächlich dazu geeignet ist, die spezifischen Probleme der wissenschaftstheoretischen Realismusdebatte in einer Weise zu beleuchten, die es erlaubt, das Patt zwischen Realisten und Antirealisten aufzulösen.

\subsection{Kants Erkenntnistheorie und die wissenschaftstheoretische Realismusdebatte}

Um Massimis Ansatz angemessen diskutieren zu können, ist es erforderlich, zunächst einen kurzen Blick darauf zu werfen, wie Massimi Kants Erkenntnistheorie darstellt. ${ }^{306} \mathrm{Ihr}$ zufolge versucht Kant dort das sog. Wissensproblem zu lösen, das sie in Form der folgenden Frage formuliert: Wie können wir sicherstellen, dass das, von dem wir glauben, dass es der Fall ist, auch tatsächlich der Fall ist? ${ }^{307}$ Dieses Problem ist keine spezifisch wis-

\footnotetext{
${ }^{302}$ Vgl. Massimi (2007), S. 239.

${ }^{303}$ Vgl. Massimi (2008), S. 2.

${ }^{304}$ Vgl. Massimi (2008), S. 5.

305 Massimis Formulierung der Wahl ist in diesem Zusammenhang, dass Phänomene nicht ,ready-made“ in der Natur vorgefunden werden, sondern in bestimmten Hinsichten ,made by us“" seien.

$306 \mathrm{Ob}$ Massimi tatsächlich eine angemessene Rekonstruktion der Position Kants liefert, ist dabei nicht Gegenstand meiner Untersuchung. Ich beschäftige mich lediglich mit der Frage, ob Massimis Argumente überzeugend sind.

${ }^{307}$ Vgl. Massimi (2008), S. 1 und 9.
} 
senschaftstheoretische Schwierigkeit, sondern betrifft all unsere Überzeugungen, auch die alltagsweltlichen. ${ }^{308}$ Kant schlägt folgende Strategie zum Umgang mit dem Wissensproblem vor: In all unseren pragmatischtechnischen Belangen interessieren wir uns für die Natur, so wie wir sie erfahren. Die Dinge, so wie sie uns erscheinen, und nicht die Dinge, wie sie an sich selbst sind, sind die Gegenstände unserer Erkenntnis. Diese werden, Kant zufolge, in wesentlichen Teilen durch unser Erkenntnisvermögen mitkonstituiert: Wir bringen Raum und Zeit als apriorische Formen unserer Sinnlichkeit sowie reine Verstandesbegriffe, die Kategorien, mit, durch die alle Empfindungsgehalte strukturiert werden. Auch alle empirischen Begriffe entstammen dem Verstand, der sie auf Grundlage der Empfindungsgehalte vermittels der Spontaneität der Einbildungskraft bildet. Verfügt jemand über empirische Begriffe, so kann er neue Empfindungsgehalte unter diese Begriffe subsumieren. Mit Hilfe der Urteilskraft werden darüber hinaus Empfindungsgehalte, die zu verschiedenen Zeitpunkten gehabt werden, miteinander verknüpft, sodass insbesondere Kausalverhältnisse theoretisch erkannt werden können. Wissen ist im Rahmen einer solchen Konzeption nicht über die Dinge, wie sie an sich selbst sind, möglich, sondern nur über die Dinge, wie wir sie im Rahmen des uns gegebenen Erkenntnisvermögens erfahren können. Wenn wir dies akzeptieren, so präsentiert Massimi die kantische Idee, höre unser Wissen auf philosophisch problematisch zu sein, da es keine generelle Kluft mehr zwischen dem, von dem wir glauben, dass es existiert, und dem, was existiert, gebe. Dass Gegenstände wie Tische und Stühle existieren, heißt dann nämlich nichts anderes, als dass sie Gegenstände der Erfahrung sind und als solche können sie nur so beschaffen sein und nur in solchen Relationen zueinander stehen, wie es die Struktur unseres Erkenntnisvermögens zulässt.

Im Folgenden wird es darum gehen, wie Massimi versucht, wesentliche Gedanken der kantischen Erkenntnistheorie zur Beantwortung wissenschaftstheoretischer Fragestellungen heranzuziehen. Deshalb soll an dieser

\footnotetext{
${ }^{308}$ In erster Annäherung sind alltagsweltliche Überzeugungen solche, die beobachtbare Gegenstände und ihre beobachtbaren Eigenschaften betreffen. Die Aussagen „Dort steht ein Tisch“ oder „Die weiße Billardkugel stößt die schwarze Kugel an“ drücken solche Überzeugungen aus. Aussagen wie „Das $\mathrm{K}^{0}$-Meson besteht aus einem Downund einem Anti-Strange-Quark“ oder „Große Massen krümmen die Raumzeit“ drücken hingegen wissenschaftliche Überzeugungen aus. In vielen Fällen zeichnen diese sich dadurch aus, dass sie uns nicht direkt in der Wahrnehmung zugänglich sind. Eine klare Abgrenzung zwischen alltagsweltlichen und wissenschaftlichen Überzeugungen ist jedoch nicht möglich.
} 
Stelle kurz diskutiert werden, wie man prima facie einen Kantianer in der wissenschaftstheoretischen Realismusdebatte verorten würde. Jemand, der Kants Erkenntnistheorie akzeptiert, ist kein Wissenschaftlicher Realist. Aber welche These des Wissenschaftlichen Realismus lehnt er ab? Auf der einen Seite erkennen Kantianer ohne weiteres an, dass es von uns unabhängige Gegenstände gibt, die unsere Sinne affizieren. Aber die Dinge, wie sie an sich selbst sind, sind uns epistemisch nicht zugänglich, wir können sie weder durch die Erfahrung erkennen noch in unseren wissenschaftlichen Theorien beschreiben. Insofern könnte man sagen, dass eine kantische Wissenschaftsphilosophie die metaphysische These des Wissenschaftlichen Realismus akzeptiert, aber den epistemologischen Optimismus des Realisten zurückweist. Andererseits besteht der Clou der kantischen Erkenntnistheorie gerade darin, dass sie eine Lösung für das Wissensproblem anbieten will. Kants Ziel ist es, aufzuzeigen, welches die Bedingungen der Möglichkeit unserer Erfahrung sind. Mithin ist Wissen für Kant ein erreichbares epistemisches Ziel. Um seine Erreichbarkeit sicherzustellen, geht Kant davon aus, dass die Gegenstände unseres Wissens keine von uns unabhängigen Dinge an sich, sondern Erscheinungen sind, die maßgeblich durch uns und unsere Verstandeskategorien geprägt werden. Vor diesem Hintergrund liegt es nahe, die kantische Auffassung so zu verstehen, dass die metaphysische These des Wissenschaftlichen Realismus (im Hinblick auf die Dinge, wie sie uns erscheinen) abgelehnt und die epistemologische These (ebenfalls im Hinblick auf die Dinge, wie sie uns erscheinen) akzeptiert wird. $^{309}$

Dies wird gestützt durch die Tatsache, dass mit Kants Theorie keine $\mathrm{Zu}-$ rückweisung einer Korrespondenztheorie der Wahrheit zugunsten eines bloß theorierelativen Wahrheitsbegriffs einhergeht (wie dies beispielsweise bei konstruktivistischen Auffassungen der Fall ist). ${ }^{310}$ Da, Kant zufolge, alle (erkenntnisfähigen) Menschen über die gleichen a priorischen Anschauungsformen und Verstandeskategorien verfügen, ist die Erscheinungswelt zwar von uns abhängig, aber dennoch objektiv und intersubjek-

\footnotetext{
${ }^{309}$ Brigitte Falkenburg nimmt eine ähnliche Zuordnung vor. Sie bezeichnet die traditionelle Position des Wissenschaftlichen Realisten als Globalen Realismus und die kantische Auffassung als Kritischen Realismus. Beide Positionen unterscheiden sich auch ihr zufolge „nur“ im Hinblick auf ihre Haltung zur metaphysischen These des Wissenschaftlichen Realismus, aber sie bleiben dennoch beide im Kern realistische Auffassungen, da für beide Wissen ein erreichbares epistemisches Ziel ist. Vgl. Falkenburg (2007), S. 13.

${ }^{310}$ Vgl. Kant ([1781/1787], 1998), A 58, B 82.
} 
tiv zugänglich. Es ist nicht beliebig oder durch idiosynkratische Faktoren (wie akzeptierte wissenschaftliche Theorien) bedingt, welche Tatsachen in der Erscheinungswelt der Fall sind. Dementsprechend sind empirische Aussagen genau dann wahr, wenn sie mit den Tatsachen (in der Welt, so wie sie uns erscheint) übereinstimmen.

12.2 Was in diesem Kapitel nicht untersucht wird und was untersucht wird

Ich stelle in diesem Kapitel nicht die generelle Frage, ob wir, wie Kant es vorschlägt, die Unabhängigkeit der Erkenntnisgegenstände vom Erkenntnissubjekt aufgeben sollten, um skeptischen Einwänden entrinnen zu können. Mir geht es nicht darum, zu entscheiden, ob wir Transzendentale Idealisten sein sollten oder nicht. Vielmehr möchte ich lediglich die Frage diskutieren, ob es spezifisch wissenschaftstheoretische Gründe gibt, die für eine kantische Konzeption naturwissenschaftlicher Phänomene sprechen. Wenn es eine korrekte Charakterisierung der kantischen Position ist, dass sie die metaphysische These des Wissenschaftlichen Realismus ablehnt, die epistemische aber akzeptiert, dann lägen solche Gründe vor, wenn die kantische Auffassung Argumente gegen die erstgenannte und/oder für die letztgenannte liefern könnte, die Vertretern anderer Auffassungen nicht zur Verfügung stehen.

Dementsprechend werde ich in Abschnitt 12.3 diskutieren, welche durch Kant zumindest inspirierten wissenschaftstheoretischen Gründe Massimi gegen die metaphysische These des Wissenschaftlichen Realismus ins Feld führt. In Abschnitt 12.4 wird dann die Frage gestellt, ob mit Hilfe der kantischen Phänomenkonzeption Argumente zur Begründung des epistemischen Optimismus im Hinblick auf unbeobachtbare Phänomene entwickelt werden können, die dem Wissenschaftlichen Realisten nicht zur Verfügung stehen. Jedoch wird mein Fazit sein, dass Massimis Antworten auf beide Fragen nicht zu überzeugen vermögen.

12.3 Gegen die metaphysische These: Massimi (und Massimis Kant) über die Genese wissenschaftlichen Wissens

Massimi vertritt die Auffassung, dass aus einer genauen Betrachtung der Art und Weise der Genese wissenschaftlicher Erkenntnisse die Schlussfol- 
gerung gezogen werden sollte, dass wissenschaftliche Phänomene nicht von uns unabhängig sind, sondern in gewissem Sinne von uns gemacht werden, da sie wesentlich durch den uns zur Verfügung stehenden Begriffsapparat geprägt werden. Massimis diesbezügliche Überlegungen nehmen ihren Ausgang von der These, dass Kant zwischen Erscheinungen und Phänomenen unterscheide. Erscheinungen seien Gegenstände der empirischen Anschauung, die raumzeitlich strukturiert, aber begrifflich noch unbestimmt seien. ${ }^{311}$ Phänomene hingegen seien Erscheinungen, die unter die Kategorien des Verstandes gebracht wurden, also begrifflich bestimmte Erscheinungen. ${ }^{312}$ Phänomene in diesem Sinne sind keine von uns unabhängigen Entitäten, weil sie als Erscheinungen bereits durch unsere Formen der Anschauung und als Phänomene zudem durch unseren Begriffsapparat geprägt seien. Die Rede von Phänomenen ist dabei selbstverständlich nicht auf wissenschaftliche Phänomene beschränkt; im Gegenteil: Alle unter Begriffe subsumierten Erscheinungen sind Phänomene und paradigmatische Fälle betreffen zuerst einmal ganz gewöhnliche Alltagserkenntnisse der Form „Die weiße Billardkugel stößt die schwarze weg.“ Massimi wendet die Unterscheidung zwischen Erscheinungen und Phänomenen auf wissenschaftliche Phänomene an, um zu zeigen, dass diese keine von uns unabhängigen Entitäten sind. Dabei präsentiert sie zwei Argumente gegen die metaphysische These des Wissenschaftlichen Realismus. Zum einen stellt sie ein Argument vor, das in sehr allgemeiner Weise die Struktur von Daten-Phänomen-Schlüssen thematisiert, und zum anderen eines, das auf einer wissenschaftshistorischen Fallstudie basiert.

\subsubsection{Die Struktur von Schlüssen von Daten auf Phänomene}

Massimis erstes Argument soll zeigen, dass sich das Modell, das Kant für unser Wissen von den Tatsachen der Alltagswelt anbietet, in einer wesent-

\footnotetext{
${ }^{311}$ Vgl. Kant ([1781/1787], 1998) A20, B34.

312 "Erscheinungen, so fern sie als Gegenstände nach der Einheit der Kategorien gedacht werden, heißen Phaenomena." Kant ([1781/1787], 1998), A249. Vgl. auch Massimi (2009), S. 9. Die exegetische Frage, ob Kant tatsächlich eine solche Unterscheidung zwischen Erscheinungen und Phänomenen vertreten hat oder ob es sich bei der zitierten Stelle um eine einzelne Formulierung ohne weitere systematische Relevanz handelt, diskutiere ich hier nicht. Mich interessiert in diesem Kapitel lediglich die Frage, ob es Massimi gelingt, diese Unterscheidung systematisch fruchtbar zu machen. Auffällig ist allerdings, dass sich die Kantstelle, auf die sich Massimi hier bezieht, nur in der A-Auflage, nicht aber in der B-Auflage der Kritik der reinen Vernunft findet.
} 
lichen Hinsicht auch auf unser Wissen von naturwissenschaftlichen Phänomenen anwenden lässt: Für beide Sorten von Wissen ist entscheidend, dass sie durch ein Wechselspiel von Sinnlichkeit und Verstand zustande kommen. Um diesen Punkt zu erläutern, skizziert Massimi die typische Struktur von Daten-Phänomen-Schlüssen: ${ }^{313}$

i) Ausgangspunkt wissenschaftlicher Untersuchungen sind Daten, die oftmals in Form relativer Häufigkeiten vorliegen. (Z.B.: Wie oft zeigt ein Teilchendetektor einen bestimmten Wert, wenn eine bestimmte Versuchsanordnung gegeben ist? Wie oft wird welche Zeit gemessen, wenn eine Bronzekugel bestimmten Gewichts eine schiefe Ebene mit einer bestimmten Länge hinabrollt?)

ii) Die Daten werden in experimentelle Parameter überführt, die als relevant erachtet werden (z.B. Streuquerschnitte beim Teilchenbeschleuniger oder die Beschleunigung der Kugeln an der schiefen Ebene).

iii) Die Daten werden in ein sog. Datenmodell überführt: ${ }^{314}$ Die experimentellen Parameter werden in einer Weise organisiert, die die Erstellung von Graphen ermöglicht, die die Abhängigkeit eines Parameters von einem anderen zeigen (z.B., wie sich der Wirkungsquerschnitt ändert, wenn die Kollisionsenergie erhöht wird, oder wie sich das Weg-Zeit-Verhältnis bei verändertem Neigungswinkel der schiefen Ebene entwickelt). Neue Phänomene können durch das Auftauchen von (unerwarteten) Graphen in Datenmodellen entdeckt werden. ${ }^{315}$

iv) Anschließend muss ein theoretisches Modell mit neuen Parametern gefunden werden, das es wahrscheinlich macht, den entsprechenden Graphen zu finden. Ursachen für das durch den Graphen repräsentierte Phänomen werden gesucht und Theorien über diese formuliert (z.B., wird die Theorie aufgestellt, dass ein bisher unbekanntes Elementarteilchen eine unerwartete Spitze des Wirkungsquerschnitts hervorgerufen hat, oder dass die Gravitations-

\footnotetext{
${ }^{313}$ Vgl. Massimi (2009), S. 10.

314 Der Begriff des Datenmodells wurde in Abschnitt 6.1.1 erläutert.

315 In Massimi (2007) wird dies anhand der Entdeckung des J/ $\psi$-Teilchens durch die Arbeitsgruppe des Physikers Burton Richter am SPEAR in Stanford illustriert. Dieses Teilchen rief eine unerwartete Spitze in einem von Richters Gruppe gemessenen Wirkungsquerschnitt hervor.
} 
kraft die Ursache dafür ist, dass die Kugeln die schiefe Ebene mit einer konstanten Beschleunigung hinabrollen).

Das strukturell in dieser Weise ablaufende Erschließen von Phänomenen auf Grundlage vorliegender Daten ist, Massimi zufolge, analog zum Übergang von Erscheinungen zu Phänomenen in Kants Erkenntnistheorie zu verstehen. ${ }^{316}$ Was sie damit meint, erläutert sie so:

"The phenomena scientists investigate are often the end product of these series
of intermediate steps, at quite distance from the original data. Not only then can
they be unobservable as Bogen and Woodward have rightly pointed out; they
may also require a significant amount of conceptual construction. By making
phenomena the serendipitous result of what Kant called the faculty of sensibil-
ity and the faculty of understanding, or-as we may prefer to say today-the re-
sult of both input from nature (in the form of data) and human contribution (in
the form of causal concepts), a Kantian stance can capture the data-to-
phenomena inference in a novel way."

Der für Massimi entscheidende Punkt scheint zu sein, dass die kantische Phänomenkonzeption der begrifflichen Konstruktionsleistung, die der Übergang von Daten zu Phänomenen erforderlich macht, besonders gut gerecht wird.

Aber, so mein Einwand, dies reicht als Begründung ihrer These, dass Phänomene keine von uns unabhängigen Entitäten sind, nicht aus. Die Anerkennung des Wechselspiels zwischen Daten auf der einen und unseren zur Interpretation der Daten in Anschlag gebrachten Begrifflichkeiten und Theorien auf der anderen Seite ist sicherlich noch kein philosophisches Alleinstellungsmerkmal. Vertreter jedweder wissenschaftstheoretischen Position erkennen an, dass es ein solches gibt. Auch Wissenschaftliche Realisten und Vertreter gängiger Antirealismen sind sich darüber einig, dass Erfahrungsurteile begrifflich strukturiert sind und wir ohne das Verfügen über bestimmte Begriffe nicht in der Lage wären, entsprechende Urteile zu fällen („Ich sehe einen DVD-Player.“), und selbstverständlich herrscht

\footnotetext{
${ }^{316}$ Vgl. Massimi (2009), S. 10. Allerdings kann damit nicht gemeint sein, dass die Daten selbst nicht begrifflich strukturiert sind. Auch Daten, also dasjenige, was beobachtet wird, sind für den Kantianer schon unter Begriffe subsumierte Erscheinungen. Sowohl Daten als auch Phänomene im Sinne Bogens und Woodwards sind demnach Phänomene im kantischen Sinne. Man darf sich hier nicht von der Äquivokation verwirren lassen.

${ }^{317}$ Massimi (2009), S. 10, Hervorhebung im Original.
} 
auch Einigkeit darüber, dass kausale Hypothesen gebildet werden, die neue Begriffe zur Erklärung von Datenmodellen einführen (schwache neutrale Ströme erklären bestimmte Spuren in der Blasenkammer, der Gravitationseinfluss eines Exoplaneten erklärt die Radialgeschwindigkeitsänderung von 51 Pegasi usw.). Wissenschaftliche Realisten sind dabei der Auffassung, dass wir durch wissenschaftliche Untersuchungen gerade herausfinden, welche Begriffe tatsächliche Strukturen in der Welt herausgreifen und welche nicht. Der Antirealist bestreitet dies (zumindest im Hinblick auf Begriffe für Unbeobachtbares). Anhand der von Massimi gegebenen Beschreibung der Struktur von Daten-Phänomen-Schlüssen lässt sich somit nicht ersehen, was eine kantische Phänomenkonzeption den konkurrierenden Auffassungen voraushätte. Will man für eine kantische Phänomenkonzeption werben, muss man aber gerade erklären, warum eine kantische Auffassung besser als andere Auffassungen dazu geeignet ist, die Struktur von Daten-Phänomen-Schlüssen zu erhellen.

\subsubsection{Galileis Untersuchung des Fallgesetzes}

Wissenschaftstheoretische Betrachtungen gewinnen ihre Überzeugungskraft häufig aus Fallstudien. Dort zeigt sich die Erklärungskraft philosophischer Theorien im Hinblick auf wesentliche Aspekte guter (oder in seltenen Fällen schlechter) wissenschaftlicher Praxis. Auch Massimi präsentiert eine solche Fallstudie, um ihre Position zu stützen. Sie wirft einen detaillierten Blick auf Galileis Untersuchung des freien Falls. Galilei gelang es, das Fallgesetz abzuleiten, obwohl er nicht in der Lage war, den freien Fall direkt zu untersuchen. Ihm fehlten für eine solche Untersuchung insbesondere hinreichend präzise Uhren. Aus diesem Grund studierte er stattdessen die gleichförmig beschleunigte Bewegung von Bronzekugeln, die eine schiefe Ebene hinabrollten, und extrapolierte seine Ergebnisse auf den freien Fall. Massimi diskutiert gerade diese wissenschaftshistorische Episode, weil auch Kant selbst immer wieder auf die Studien und Experimente Galileis Bezug nimmt. Galilei ist für Kant einer der Wegbereiter einer neuen Form Naturwissenschaft zu betreiben. So schreibt Kant in der Vorrede zur zweiten Auflage der Kritik der reinen Vernunft:

„Als Galilei seine Kugeln die schiefe Fläche mit einer von ihm selbst gewählten Schwere herabrollen ließ, oder Torricelli die Luft ein Gewicht, was er sich zum voraus dem einer ihm bekannten Wassersäule gleich gedacht hatte, tragen ließ, oder in noch späterer Zeit Stahl Metalle in Kalk und diesen wiederum in Metall 
verwandelte, indem er ihnen etwas entzog und wiedergab [...]; so ging allen Naturforschern ein Licht auf. Sie begriffen, daß die Vernunft nur das einsieht, was sie selbst nach ihrem Entwurfe hervorbringt, daß sie mit Prinzipien ihrer Urteile nach beständigen Gesetzen vorangehen und die Natur nötigen müsse auf ihre Fragen zu antworten, nicht aber sich von ihr allein gleichsam am Leitbande gängeln lassen müsse; denn sonst hängen zufällige, nach keinem vorher entworfenem Plane gemachten Beobachtungen gar nicht in einem notwendigen Gesetze zusammen, welches doch die Vernunft sucht und bedarf. Die Vernunft muss mit ihren Prinzipien, nach denen allein übereinkommende Erscheinungen für Gesetze gelten können, in einer Hand, und mit dem Experiment, das sie sich nach jenen ausdachte, in der anderen, an die Natur gehen, zwar um von ihr belehrt zu werden, aber nicht in der Qualität eines Schülers, der sich alles vorsagen läßt, was der Lehrer will, sondern eines bestallten Richters, der die Zeugen nötigt auf die Fragen zu antworten, die er ihnen vorlegt. Und so hat sogar die Physik die so vorteilhafte Revolution ihrer Denkart lediglich dem Einfalle zu verdanken, demjenigen was die Vernunft selbst in die Natur hineinlegt, gemäß, dasjenige in ihr zu suchen, (nicht ihr anzudichten,) was sie von dieser lernen muß, und wovon sie für sich selbst nichts wissen würde. Hierdurch ist die Naturwissenschaft allererst in den sicheren Gang einer Wissenschaft gebracht worden, da sie so viele Jahrhunderte durch nichts weiter als bloßes Herumtappen gewesen war.، ${ }^{6318}$

Kant betont hier die zentrale Bedeutung des Wechselspiels zwischen externer Wirklichkeit, in Form der Ergebnisse von Experimenten, und begrifflicher Konstruktionsleistung gemäß der Prinzipien den Vernunft. Er behauptet sogar, dass die Vernunft nur das einsehen könne, was sie nach ihrem eigenen Entwurf hervorbringe, was sie selbst in die Natur „hineinlegt“..${ }^{319}$ Erst hierdurch werde die Naturwissenschaft in den sicheren Gang einer wirklichen Wissenschaft gebracht. Dieser Punkt ist, Kant zufolge, wesentlich für das Verständnis von Galileis Untersuchung des freien Falls. Massimi teilt diese Auffassung. Dabei interpretiert sie die gerade zitierte Stelle in der folgenden Weise:

„Galileo started indeed with appearances, namely with observed relative motions of heavy bodies, whose kinematics he carefully studied. For the sake of experience, he inserted something a priori into these appearances: namely he took those relative motions as approximating to uniformly accelerated motions

\footnotetext{
${ }^{318}$ Kant ([1781/1787], 1998) B XII-XIV

319 Ich benutze im Folgenden häufiger diese Formulierung, die sich bei Kant und Massimi (,to insert“) findet. Allerdings nicht etwa, weil ich sie für besonders klar hielte, sondern, im Gegenteil, weil mir nicht ganz klar ist, was genau gemeint ist und ich deshalb nicht in der Lage bin, eine geeignetere Formulierung vorzuschlagen.
} 
due to a moving force. Finally, with the experiment of the inclined plane, he extracted and demonstrated what he had previously inserted into appearances for the sake of possible experience, namely uniformly accelerated motions with gravitational attraction." ${ }^{320}$

Das Ziel der Experimente mit der schiefen Ebene bestand dieser Interpretation zufolge darin, aus den Erscheinungen (Bewegung eines Bronzeballs auf der schiefen Ebene) die Eigenschaft einer durch eine Kraft verursachten gleichförmigen Beschleunigung zu extrahieren, welche Galileo zuvor $a$ priori in die Erscheinungen hineingelegt habe, um die Erfahrung einer Bewegung diesen Typs erst möglich zu machen. ${ }^{321}$ Hier irgendwo liegt das konstruktive Element, das die Ablehnung der metaphysischen These erforderlich machen soll. Doch worin genau besteht es? Massimi versucht aufzuzeigen, dass ohne die Anerkennung bestimmter, nicht weiter begründeter Aussagen als wahr, Galilei nicht in der Lage gewesen wäre, das Fallgesetz abzuleiten. Dies zeige sich in den Discorsi, wo Galilei sein Vorgehen beschreibt. Dort berichtet Salviati (d.i. diejenige Figur, deren Äußerungen Galileis Standpunkt repräsentieren), dass Galilei eine wichtige Voraussetzung machen musste, um die Korrektheit des Fallgesetzes beweisen zu können. Diese Voraussetzung (im weiteren Verlauf abgekürzt mit dem Buchstaben V) lautet:

(V) „Die Geschwindigkeitswerte, welche ein und derselbe Körper bei verschiedenen Neigungen einer Ebene erlangt, sind einander gleich, wenn die Höhen dieser Ebenen gleich sind.“' ${ }^{\text {322 }}$

Mit Hilfe von V gelingt es Galilei, das Fallgesetz abzuleiten. ${ }^{323}$ Aber Sagredo (einer der beiden Gesprächspartner Salviatis) ist nicht, zumindest nicht völlig, von der Wahrheit von V überzeugt. Um ihn endgültig zu überzeugen, präsentiert Salviati ein Gedankenexperiment, das durch Abbildung 5 erläutert wird und folgende Überlegung beinhaltet: Man nehme an, dieses Blatt sei eine in der Horizontalebene errichtete Wand. An der Stelle A sei ein Nagel in die Wand geschlagen, an dem ein Faden befestigt ist. Am Ende des Fadens hängt eine Bleikugel. Der Faden sei gespannt und die Kugel befinde sich am Punkt $\mathrm{C}$.

\footnotetext{
${ }^{320}$ Massimi (2008), S. 17, meine Hervorhebung. Vgl. auch Massimi (2009), S. 11.

${ }^{321}$ Vgl. Massimi (2009), S. 11-12.

${ }^{322}$ Galilei ([1638] 1987), S. 384.

${ }^{323}$ Vgl. Galilei ([1638] 1987), S. 389-390.
} 


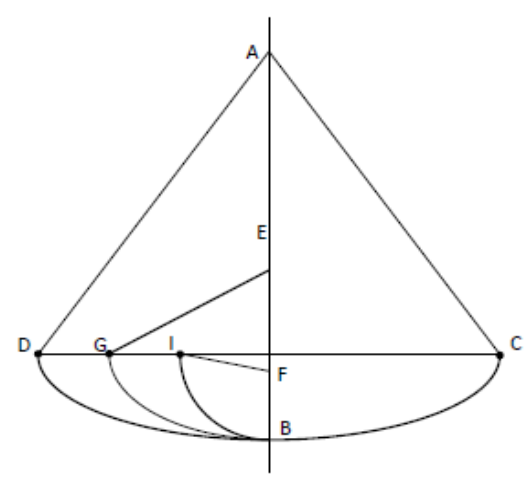

Abbildung 5: Galileis Gedankenexperiment (nach Galilei ([1638], 1987), S. 386)

Wenn man die Kugel loslässt, so wird sie sich (unter der Vernachlässigung des Luftwiderstands) über B zum Punkt D bewegen. Wenn man jedoch im Punkt E einen weiteren Nagel befestigt, der die Bewegung des oberen Teils des Fadens über diesen Punkt hinaus verhindert, so wird die Geschwindigkeit der Kugel im Punkt B ausreichen, um sie bis zum Punkt G zu befördern (Analoges gilt für F und I). Galilei folgert hieraus:

„Denn da die Bögen CB, DB einander gleich sind und symmetrisch liegen, so wird das beim Sinken durch den Bogen CB erlangte Moment ebenso groß sein, wie die Wirkung durch den Bogen DB; aber das in B erlangte, durch CB hindurch erzeugte Moment, vermag denselben Körper durch den Bogen BD zu heben; folglich wird auch das beim Fallen durch DB hervorgerufene Moment gleich sein demjenigen, welches den selben Körper vorher von B bis D zu fördern vermochte, so daß allgemein jedes beim Fallen erzeugte Moment gleich demjenigen ist, welches der Körper durch denselben Bogen zu erheben imstande ist: aber alle Momente, die den Körper durch die Bögen BD, BG, BJ zu heben vermochten, sind einander gleich, da sie stets durch das Fallen durch CB entstanden waren, wie der Versuch es lehrt: folglich sind auch die Momente, die durch die Bögen DB, GB, JB hervorgerufen werden, einander gleich.“(324

Diese Schlussfolgerung überträgt er im Anschluss von Bögen wie CB (also gekrümmten Flächen) auf die Sehnen der Bögen (also schiefe Ebenen): Wenn wir die Kugel, statt sie an einem Faden schwingen zu lassen, auf einer schiefen Ebene von $C$ nach $B$ rollen lassen, so verfügt die Kugel im Punkt B über ein hinreichend großes Moment (der Ausdruck „Moment" bezeichnet in etwa das, was wir heute als Impuls bezeichnen ${ }^{325}$ ), um eine

\footnotetext{
${ }^{324}$ Galilei ([1638] 1987), S. 386-387.

${ }^{325}$ Vgl. Fischer (1992), S. 178 zum Verhältnis zwischen Galileis Moment- und dem Impulsbegriff der Newton'schen Mechanik.
} 
schiefe Ebene von B nach D, G oder I hinaufzulaufen. ${ }^{326}$ Folglich müsse die Kugel beim Hinabrollen solcher Ebenen jeweils ein gleich großes Moment bekommen und damit die gleiche Endgeschwindigkeit erreichen. Mit dieser Überlegung meint Galilei einen „,fast zwingenden Beweis ${ }^{\text {‘327 }}$ der oben beschriebenen Voraussetzung V geliefert zu haben. Die Argumentation sei noch nicht völlig zwingend, da der Übergang von gekrümmten zu ebenen Flächen einen inferentiellen Sprung beinhalte. Erst das Experiment könne zeigen, ob dieser Übergang legitim ist:

„Wollen wir dies nunmehr als Postulat gelten lassen; die absolute Richtigkeit wird uns später einleuchten, wenn wir die Folgerungen aus solcher Hypothese eintreffen und genau mit dem Versuch übereinstimmen sehen.“3228

Im Dialog lässt sich Sagredo durch das Gedankenexperiment überzeugen und akzeptiert die entsprechende Ableitung des Fallgesetzes, aber Simplicio, der Gesprächspartner der beiden, der den aristotelischen Standpunkt repräsentiert, fordert experimentelle Belege für die aufgestellten Hypothesen. Salviati referiert daraufhin die Ergebnisse von Galileis Experimenten an der schiefen Ebene, auf die auch Kant in seiner Vorrede Bezug nimmt. $^{329}$

Inwiefern kann nun anhand dieser Beschreibung der Vorgehensweise Galileis Massimis These gestützt werden, dass es in ihr ein apriorisches Element gebe, das das Einnehmen des kantischen Standpunktes erforderlich mache? Massimi beantwortet diese Frage so:

„To sum up, Kant suggested a radically new conception of phenomena, according to which a phenomenon, say the phenomenon of a uniformly accelerated free-falling object, is something that from the very outset we have mathematically-geometrically constituted as an object having certain spatiotemporal properties [...] and, most importantly, subsumed under a causal concept by tracing those spatiotemporal properties back to some moving force $[\ldots]$

In this specific sense, Galileo exemplified Kant's Copernican turn by showing how phenomena that scientists investigate are not ready-made for us to either save them or give a literally true story of them, but instead they have build in

\footnotetext{
${ }^{326}$ Praktisch funktioniert dies natürlich aufgrund des entstehenden Knicks im Punkt B nicht.

${ }^{327}$ Galilei ([1638] 1987), S. 385.

${ }^{328}$ Galilei ([1638] 1987), S. 387-388.

${ }^{329}$ Vgl. Galilei ([1638] 1987), S. 23.
} 
them some a priori elements that we have to extract and prove through experiment." $" 330$

Das apriorische Element, das für Galileis Etablierung des Fallgesetzes eine Rolle spielt, ist die Begründung der Voraussetzung V. ${ }^{331}$ Dabei ist nicht so sehr entscheidend, dass es sich bei dieser Begründung um ein Gedankenexperiment handelt, sondern dass der Übergang von dem im Gedankenexperiment geschilderten Szenario zur schiefen Ebene durch die Einführung einer theoretischen Größe erfolgt: Galilei spricht von gleich großen Momenten. Ein Moment wiederum ist Ausdruck einer Bewegungskraft: Durch die Einwirkung einer Kraft wird das Moment eines Körpers verändert. Im Rahmen seiner Argumentation muss Galilei demnach, um die Richtigkeit von $\mathrm{V}$ zu zeigen, a priori eine kausale Begrifflichkeit einführen. ${ }^{332}$ So jedenfalls Massimi.

Angenommen, die vorhergehende Darstellung ist eine angemessene Rekonstruktion von Massimis These, dass wissenschaftliche Phänomene keine von uns unabhängigen Sachverhalte sind, ist ihre Begründung überzeugend? Massimi behauptet, dass ohne eine apriorische Annahme die Erfahrung einer gleichförmig beschleunigten Bewegung aufgrund einer Kraft nicht möglich gewesen sei (,For the sake of experience, he inserted something a priori into these appearances: namely he took those relative motions as approximating to uniformly accelerated motions due to a moving force. ${ }^{6333}$ ). Dies wiederum begründet sie so:

Namely, for the sake of experiencing uniformly accelerated motion, we must constitute the properties of free-falling bodies according to Galileo's kinematical reasoning - Galileo did not arrive at his law of free fall by simple curvefitting data about balls rolling down inclined planes. There was instead an element of construction, a "principle of reason" that guided Galileo in his experiments with inclined planes and led him to the phenomenon of uniformly accelerated free-falling bodies." 334

Wie ist dies zu verstehen? Will Massimi nur behaupten, dass Galilei eine kausale Hypothese der Form „Es gibt eine Bewegungskraft, die die Ursa-

${ }^{330}$ Massimi (2008), S. 27-28, Hervorhebungen im Original. Vgl. auch Massimi (2009), S. 9.

${ }^{331}$ Vgl. Massimi (2008), S 21-28, insb. S. 26.

332 A priori ist die Annahme der Bewegungskraft wohl deshalb, weil ihr Begriff gebildet wird bevor die entsprechenden Erfahrungen gemacht werden können.

${ }^{333}$ Massimi (2008), S. 17, meine Hervorhebung.

${ }^{334}$ Massimi (2009), S. 11-12. 
che dafür ist, dass Körper gleichförmig beschleunigt fallen" aufgestellt hat, welche ihn bei der Konstruktion seines Experiments leitet, und von der sich im Verlauf seiner empirischen Untersuchungen herausstellte, dass sie durch die experimentellen Ergebnisse bestätigt wird? Wenn dem so wäre, wäre ihre These philosophisch nicht besonders interessant. Sie bestünde dann in der schlichten Feststellung, dass Wissenschaftler häufig bereits im Vorfeld Hypothesen über Phänomene und deren Ursachen formulieren, die sie experimentell untersuchen, und dass die Konstruktion von Experimenten oft durch diese Hypothesen geleitet wird. Manchmal werden Erwartungen der Wissenschaftler experimentell bestätigt, manchmal enttäuscht. Galilei gehörte zu den Glücklichen, deren Hypothese bestätigt wurde. Dieses unbestreitbare Faktum spricht allerdings weder für noch gegen irgendeine wissenschaftstheoretische Auffassung, es ist vielmehr problemlos mit unterschiedlichen wissenschaftstheoretischen Positionen vereinbar und liefert somit keinen Grund für die These, dass Kants Erkenntnistheorie eine bislang unberücksichtigte weitere Option in der Realismusdebatte motivieren könne.

Da es ihr Ziel ist, eine solche neue Theorieoption aufzuzeigen, muss Massimi eine stärkere These vertreten. Sie muss die Formulierung „for the sake of experience" ernst meinen, d.h. behaupten, dass wir die Bewegung ohne Annahme einer Bewegungskraft auch nicht als gleichförmig beschleunigt erfahren können. Aber diese stärkere Behauptung ist meines Erachtens nicht haltbar. Das Fallgesetz selbst lässt sich in rein kinematischen Begrifflichkeiten (Strecke, Zeit und Beschleunigung) formulieren, ohne den Begriff einer Kraft zu verwenden. Warum sollte man seine Richtigkeit nicht durch Experimente an der schiefen Ebene und „simple Kurvenanpassung“ überprüfen können? ${ }^{335}$ Letztlich tat dies auch Galilei, selbst wenn er zusätzlich noch Vermutungen über die Ursache der gleichförmig beschleunigten Fallbewegung anstellte und die Wahrheit seiner Vermutungen für a priori einsehbar hielt. Natürlich gibt es in Galileis Überlegungen einen inferentiellen Sprung, nämlich bei der Extrapolation von der schiefen Ebene auf den freien Fall. Aber dieser Sprung wird nicht durch die Annahme einer konstanten Bewegungskraft erst möglich gemacht, sondern das Abhängigkeitsverhältnis ist genau umgekehrt: Nur wenn diese Extrapolation legitim ist, darf eine konstante Bewegungskraft angenommen werden. ${ }^{336}$

${ }^{335}$ Ich sehe hier zunächst vom Unterbestimmtheitsproblem ab, das im vorherigen Kapitel diskutiert wurde. Später wird es jedoch eine Rolle spielen.

${ }^{336}$ Und die Legitimität der Extrapolation ist prinzipiell empirisch prüfbar. Galileis Uhren waren vielleicht nicht genau genug. Aber heute sind wir in der Lage Fallexperi- 
Somit ist nicht zu sehen, warum ein Rekurs auf kausale Begriffe wie den der Anziehungskraft notwendig ist, um das Phänomen der gleichförmig beschleunigten Bewegung aus den Daten erschließen zu können. ${ }^{337}$

Somit bleibt festzuhalten: Die Kantianerin mag den Punkt machen, dass wir uns die Welt unter Rückgriff auf bestimmte Begriffe, insbesondere den Kausalitätsbegriff, erschließen. Wir begreifen verschiedene Ereignisse als kausal verknüpft und entwickeln Theorien, die unbeobachtbare Ursachen für beobachtete Wirkungen beschreiben. Dies mag des Weiteren daran liegen, dass Kausalität eine Kategorie unseres Verstandes ist und wir deshalb gar nicht anders können, als die uns erscheinende Welt dieser Kategorie entsprechend zu konstruieren. Aber: Wenn es für diese Auffassung Gründe gibt, liegen sie in Kants allgemeiner Erkenntnistheorie und nicht in seiner Wissenschaftstheorie. Die wissenschaftliche Praxis (in Gestalt von Galileis Experimenten an der schiefen Ebene) liefert hierfür keine zusätzlichen Gründe.

\subsection{Für die epistemische These: Massimi (und Kant) über unbeobachtbare Phänomene}

Das Anliegen von Kants Erkenntnistheorie lässt sich im Hinblick auf die Sphäre unseres Alltagswissens, d.h. unser Wissen von beobachtbaren Gegenständen und ihren beobachtbaren Eigenschaften, so formulieren: Kant versucht, durch Aufgabe der metaphysischen These die epistemische These zu retten. Im Folgenden gilt es, zu untersuchen, ob man im Hinblick auf unbeobachtbare wissenschaftliche Phänomene vielleicht ein ähnliches Manöver vornehmen kann.

mente im Vakuum durchzuführen und die relevanten kinematischen Größen zu messen. So können wir das Fallgesetz ohne den Umweg über die schiefe Ebene bestätigen. Darüber hinaus wissen wir heute, dass die Kraft nur in Annäherung konstant wirkt, tatsächlich aber vom Abstand des fallenden Körpers von der Erdoberfläche abhängt.

${ }^{337}$ Dies gilt insbesondere, da Galilei in seinen früheren Studien, die er in De Motu beschreibt, noch ganz andere Prinzipien als klare und selbstevidente Voraussetzungen anerkannte, diese aber später verwerfen musste, weil sich Folgerungen aus diesen nicht experimentell bestätigen ließen. (Galilei glaubt hier noch, dass Gegenstände höherer Dichte schneller fallen als Gegenstände geringerer Dichte.) Auch hier gab er also der Empirie das letzte Wort. Vgl. Fischer (1992), S. 177. 
12.4.1 Epistemischer Optimismus aufgrund apriorischer Einsichten?

Im Bereich wissenschaftlicher Erkenntnisse, die uns nicht direkt in der Erfahrung, sondern nur über Theorien vermittelt zugänglich sind, geschieht, Massimi zufolge, nichts genuin anderes als im Bereich der alltagsweltlichen Erkenntnis. Die unbeobachtbaren Phänomene, von denen wissenschaftliche Theorien handeln, hängen zwar wesentlich von uns ab; sie sind zumindest teilweise unsere Konstrukte, aber dies gilt eben auch für die Gegenstände der Erscheinungswelt, die wir mit bloßem Auge beobachten können. Insofern stehen beobachtbare und unbeobachtbare Phänomene ontologisch und epistemologisch auf der gleichen Ebene: Beide gehören zum ontologischen Inventar der Welt der Erscheinungen, die wir (prinzipiell) erkennen können. ${ }^{338}$ In einem früheren Aufsatz spricht Massimi deshalb auch davon, dass ihre Position eine moderate kantische Form des Realismus sei. ${ }^{339}$ In den hier thematisierten Aufsätzen verwendet sie zwar nicht mehr diese Formulierung, aber dennoch ist meines Erachtens klar erkennbar, dass sie nach wie vor eine entsprechende Idee verfolgt. So betont sie immer wieder, dass ihrer kantischen Konzeption zufolge Phänomene zwar keine von uns (vollständig) unabhängigen Entitäten seien, aber dies nicht dazu verleiten dürfe, konstruktivistische, empiristische oder konventionalistische Positionen $\mathrm{zu}$ beziehen. ${ }^{340}$ Sie grenzt sich beispielsweise von Duhem ab, der schreibt

\footnotetext{
${ }^{338}$ Ist es nicht von vornherein widersprüchlich, unbeobachtbare Gegenstände als Gegenstände in der Welt der Erscheinungen zu verorten? Ich denke nicht, denn Kant geht nicht davon aus, dass das, was wir erfahren oder wahrnehmen, unsere eigenen Ideen, Eindrücke oder Sinnesdaten sind, sondern Gegenstände außerhalb unserer selbst. Nur dasjenige, was wir von diesen Gegenständen wissen können, ist maßgeblich von der Struktur unseres Erkenntnisvermögens abhängig. In genau diesem Sinne behauptet Kant auch, dass bestimmte unbeobachtbare Entitäten, die naturwissenschaftliche Theorien postulieren, als existierend anzuerkennen sind. Vgl. hierzu das Kantzitat in Abschnitt 6.2.

${ }^{339}$ Massimi (2007), S. 240. In diesem Aufsatz geht es Massimi hauptsächlich um eine Kritik an van Fraassens Position; ihre eigene Auffassung deutet sie dort hingegen nur an. Deshalb behandele ich diesen Text hier nicht ausführlicher.

${ }^{340}$ Vgl. auch Massimi (2008), S. 4 FN 2, S. 16, S. 25, S. 35, Massimi (2009), S. 11-15.
} 
„Despite Kepler and Galileo, we believe today, with Osiander and Bellarmine, that the hypotheses of physics are mere mathematical contrivances devised for the purpose of saving the phenomena" ${ }^{341}$,

indem sie ihm Folgendes entgegenhält

„Kant's new conception of phenomena is indeed patterned up on Galileo's in believing that scientific investigation of nature can reveal its inner lawfulness, and therefore that the gap between what we believe there is and what there $i s$, is not as big as Bellarmine and his followers claimed. ${ }^{, 342}$

Aufgrund der hier und an zahlreichen anderen Stellen vorgenommenen Distanzierung von gängigen Antirealismen halte ich es für gerechtfertigt, die Position, die Massimi begründen möchte, weiterhin als kantischen Realismus $\mathrm{zu}$ bezeichnen, auch wenn sich diese Formulierung in ihren aktuellsten Texten nicht mehr findet. Ein solcher realistischer Anspruch deckt sich darüber hinaus mit Kants eigenen philosophischen Zielen. In der Schrift Metaphysische Anfangsgründe der Naturwissenschaft verspricht Kant, die apodiktische Gewissheit der Gesetze der Physik aufzuzeigen. ${ }^{343}$ Dies bedeutet, wie Brigitte Falkenburg erläutert, dass nach Kants Erkenntnislehre die Gesetze der Physik ,durch die metaphysische Begründung mit Notwendigkeit als wahr erkannt werden sollen." ${ }^{634}$ Mit anderen Worten: Kant möchte eine metaphysische Begründung der epistemischen These des Wissenschaftlichen Realismus liefern.

Wie stellt sich Kant eine solche Begründung vor? In den Metaphysischen Anfangsgründen der Naturwissenschaft bestimmt Kant die Materie als dasjenige, was in einem leeren Raum beweglich ist. Durch zwei Grundkräfte, eine Anziehungs- und eine Zurückstoßungskraft, soll das gesamte Verhal-

${ }^{341}$ Duhem (1969), S. 117. Man beachte, dass Duhem mit dem Ausdruck „Phänomene“ bzw. ,phenomena“ ausschließlich beobachtbare Prozesse und Strukturen bezeichnet. Osiander ist der Verfasser des Vorwortes von Kopernikus' De Revolutionibus Orbium Coelestium. Dort erklärt er, dass Kopernikus“ Theorie als bloß nützliches Rechenmodell anzusehen sei, aber nicht den Anspruch habe, die Wahrheit des heliozentrischen Weltbilds zu propagieren. Bellarmine war Bischof und gleichzeitig ein enger Freund Galileis, der diesem auch die Haltung der katholischen Kirche gegenüber Kopernikus` Schriften vermittelte. Auch Bellarmine war der Auffassung, dass Kopernikus` Theorie instrumentalistisch interpretiert werden müsse.

${ }^{342}$ Massimi (2008), S. 8, Hervorhebungen im Original.

${ }^{343}$ Vgl. Kant ([1786] 1997), AA IV 469.

${ }^{344}$ Falkenburg (2000), S. 335, Hervorhebung im Original. 
ten der Materie erklärt werden. ${ }^{345}$ Dass es solche Kräfte geben muss, können wir Kant zufolge a priori einsehen, denn nur durch das Vorhandensein solcher Kräfte ist es der Materie möglich, einen zuvor leeren Raum zu erfüllen. Unser Begriff der Materie präsupponiert deshalb den Kraftbegriff. Diese Integration kausaler Begriffe in unsere Begriffe für Beobachtbares ist, laut Massimi, das entscheidende Novum eines kantischen Phänomenverständnisses:

„I think this is the crucial, distinctively new feature that Kant introduced into the conception of phenomena: a physical phenomenon - intended as a conceptually determined appearance - has built in it from the very outset the concept of a moving force as the cause of the observed appearance. It is the causal concept of a moving force that distinguishes phenomena from appearances, or better, that transforms appearances into phenomena; i.e. objects of possible experience into objects of experience." $" 346$

Diese Textpassage, insbesondere die These des Eingebaut-Seins kausaler Begriffe in physikalische Phänomene, ist nicht leicht zu verstehen. Gemeint sein könnte: Als Gegenstände der Erfahrung werden Phänomene immer schon als in Kausalzusammenhängen stehend begriffen. Wir können unsere Erfahrungsinhalte nicht von kausalen Begriffen, unter die wir jene subsumieren, ablösen und getrennt behandeln. In den Begriff des Phänomens des freien Falls beispielsweise ist auf irgendeine Weise der Begriff einer diese Bewegung verursachenden Kraft bereits integriert. Alle Gegenstände der Erfahrung unterliegen kausalen Gesetzmäßigkeiten und wir können a priori einsehen, dass es Ursachen bestimmter Art geben muss, damit die Erscheinungen so sind, wie sie sind. Natürlich behaupten Kant und Massimi nicht, dass wir a priori wissen könnten, wie die Gravitationskraft quantitativ beschaffen ist, also, dass wir das Newton'sche Gravitationsgesetz a priori ableiten können. Welche quantitativen kausalen Gesetzmäßigkeiten gelten, müssen die Wissenschaftler in sorgfältigen experimentellen Studien herausfinden. Aber, so könnte man Massimi verstehen, wir wissen immerhin a priori, dass es eine Ursache des freien Falls in Form einer Kraft geben muss. Wenn wir experimentell die quantitative Beschaffenheit dieser Kraft studiert haben, können wir ein Naturgesetz formulieren, das Eigenschaften dieser Kraft angibt, und können einsehen, dass

\footnotetext{
${ }^{345}$ Vgl. Kant ([1786] 1997), AA IV 496-498. Kant selbst sah später ein, dass allein mit diesen zwei Kräften nicht alle empirischen Eigenschaften der Materie erklärbar waren. Massimi (2008), S. 13-14.

${ }^{346}$ Massimi (2008), S. 14, Hervorhebungen im Original.
} 
es mit Notwendigkeit wahr sein muss. Die beschriebenen Kräfte wären dabei deshalb die tatsächlichen Kräfte, weil sie den Gesetzmäßigkeiten unseres Denkens entspringen, die wir wiederum der Natur vorschreiben. Wenn dies stimmen und für alle wissenschaftlichen Phänomene gelten würde, so könnte vielleicht verständlich gemacht werden, weshalb wir nicht an der Existenz der unbeobachtbaren Entitäten unserer besten Theorien zweifeln sollten. Ihre Existenz ist durch die Existenz beobachtbarer Gegenstände gesichert, denn insofern diese Gegenstände unserer unmittelbaren Erfahrung sind, muss es Ursachen für ihr Verhalten geben. So wie wir a priori einsehen können, dass der freie Fall durch eine Gravitationskraft verursacht wird, könnten wir dann a priori einsehen, dass andere Erscheinungen durch Ursachen in der Form des Verhaltens anderer unbeobachtbarer Entitäten hervorgebracht werden.

Aber eine solche Auffassung wäre eine unplausible ad-hoc-Lösung des Problems. Die Schwierigkeit, wahre von bloß instrumentell nützlichen Hypothesen zu unterscheiden, würde wegerklärt, indem man die Annahme trifft, dass wir aufgrund einer apriorischen Einsicht in die Prinzipien der Vernunft wissen können, welche Hypothesen die richtigen sind. Dies ist nicht überzeugend, weil es an dieser Stelle um etwas viel Spezifischeres geht, als darum, ob es eine allgemeine Eigenschaft unseres Erkenntnisvermögens ist, Ereignisse als kausal verknüpft zu begreifen (was der Fall sein mag oder auch nicht); nämlich darum, ob die Ursache des freien Falls eine Gravitationskraft, das Strebevermögen eines Körpers hin zu seinem ,natürlichen Platz" im Universum oder eine Krümmung der Raumzeit ist. Warum sollte man a priori einsehen können, dass eine dieser möglichen Ursachen die richtige ist? Diese Frage drängt sich vor dem Hintergrund der kantischen Erkenntnistheorie auch deswegen auf, weil die Theorien, deren apodiktische Gewissheit Kant a priori demonstrieren wollte, heute als falsifiziert gelten. Auch ein Vertreter der Allgemeinen Relativitätstheorie ist in der Lage aus den Gleichungen seiner Theorie das Fallgesetz abzuleiten. Aber, wenn die Allgemeine Relativitätstheorie wahr ist, dann ist Gravitation ein Phänomen, das durch Krümmungen der Raumzeit selbst zustande kommt, und kein Phänomen in der Raumzeit. Und vielleicht gibt es, so wie es die These von der empirischen Unterbestimmtheit besagt, noch zahlreiche andere, bisher noch nicht erdachte Theorien, die eine entsprechende Ableitung ermöglichen. Wenn sich überzeugend für ein solches Unterbestimmtheitsszenario argumentieren lässt (und dies behaupten, wie wir sahen, zahlreiche Kritiker des Wissenschaftlichen Realismus), dann stellt sich für den Kantianer die gleiche Frage, die sich für den Wissenschaftli- 
chen Realisten stellt, nämlich, weshalb wir davon ausgehen sollten, dass unsere Theorien auch die „richtigen“ Entitäten postulieren. ${ }^{347}$

12.4.2 Worum es in der wissenschaftstheoretischen Realismusdebatte eigentlich geht und weshalb Massimi und Kant hierzu nichts beitragen

Die Begründung des epistemischen Optimismus vermittels apriorischer Einsichten vermag nicht zu überzeugen und eine andere Begründung spezifisch kantischer Art kann ich in Massimis Texten nicht finden. Aber die Suche nach einer solchen Begründung ist es, worum sich die Debatte um den Wissenschaftlichen Realismus dreht: Es geht dort um die Frage, ob wir den theoretischen Aussagen der Wissenschaft den gleichen erkenntnistheoretischen Status zusprechen sollten wie Aussagen über beobachtbare Gegenstände.

Wie bereits beschrieben, stellt sich diese Frage auch für den Kantianer: Epistemisch zugänglich ist uns, ihm zufolge, zwar nur die Erscheinung der Dinge, die durch unsere Anschauungsformen und Verstandeskategorien strukturiert ist. Dennoch ist diese Erscheinungswelt objektiv insofern, dass alle Menschen über die gleichen Verstandeskategorien und Anschauungsformen verfügen. Der Unterschied zwischen kantischem und Wissenschaftlichem Realismus betrifft somit „bloß“ den ontologischen Status von Gegenständen schlechthin und hat keine spezifisch wissenschaftstheoretische Dimension. Diese Dimension ergibt sich daraus, dass die kausalen Begriffe der Wissenschaften spezifische Begriffe einzelner Theorien (z.B. der Begriff der Gravitationskraft, des Atoms, des Moleküls, des Gens, des Charmquarks o.ä.) sind und dass wir zu den durch diese Begriffe (vermeintlich) bezeichneten Entitäten nur auf inferentiellem Wege Zugang haben. Auch einen Kantianer kann man im Hinblick auf solche Entitäten mit dem Unterbestimmtheitsargument oder der pessimistischen Metainduktion konfrontieren und die Frage aufwerfen, ob die Ausdrücke „Atom“ und „Molekül“ ebenso Gegenstände in der Erscheinungswelt bezeichnen wie „Tisch“ und „Tannenbaum“. Dass sich diese Fragen auch für den Kantianer stellen, wird zudem deutlich, wenn man Kants eigene Ausführungen betrachtet, die ausdrücklich den theoretischen Entitäten der Wissenschaft gewidmet sind:

${ }^{347}$ Massimi (2008), S. 27 zeigt an, dass Massimi sich dieses Problems durchaus bewusst ist. 


\begin{abstract}
„Man kann aber auch vor der Wahrnehmung des Dinges, und also comparative a priori das Dasein desselben erkennen, wenn es nur mit einigen Wahrnehmungen, nach den Grundsätzen der empirischen Verknüpfung derselben (den Analogien), zusammenhängt. Denn alsdenn hängt doch das Dasein des Dinges mit unseren Wahrnehmungen in einer möglichen Erfahrung zusammen, und wir können nach dem Leitfaden jener Analogien, von unserer wirklichen Wahrnehmung zu dem Dinge in einer Reihe möglicher Wahrnehmungen gelangen. So erkennen wir das Dasein einer alle Körper durchdringenden magnetischen Materie aus der Wahrnehmung des gezogenen Eisenfiligs, obzwar eine unmittelbare Wahrnehmung dieses Stoffs uns nach der Beschaffenheit unserer Organe unmöglich ist. Denn überhaupt würden wir, den Gesetzen der Sinnlichkeit und dem Kontext unserer Wahrnehmungen, in einer Erfahrung auch auf die unmittelbare empirische Anschauung derselben stoßen, wenn unsere Sinnen feiner wären, deren Grobheit die Form möglicher Erfahrung überhaupt nichts angeht. Wo also Wahrnehmung und deren Anhang nach empirischen Gesetzen hinreicht, dahin reicht auch unsere Erkenntnis vom Dasein der Dinge. “348
\end{abstract}

Kant vertritt hier offensichtlich eine moderate Version des Realismus, d.h. die These, dass die theoretischen Entitäten wissenschaftlicher Theorien den gleichen epistemologischen Status haben wie beobachtbare Entitäten. Kants Argument hierfür besteht im Vertrauen auf die Verlässlichkeit der wissenschaftlichen Methodik, in deren Rahmen auf die Existenz dieser Entitäten geschlossen wird. ${ }^{349}$ Für Kant ist nur die Beschränktheit unserer Sinnesorgane dafür verantwortlich, dass uns die entsprechenden Gegenstände nicht direkt in der Wahrnehmung zugänglich sind.

Kant kannte allerdings noch nicht die Einwände, die beispielsweise Duhem, Kuhn, Feyerabend, Quine, Laudan, van Fraassen oder Stanford gegen den Wissenschaftlichen Realismus vorgebracht haben. Insbesondere war Kant davon überzeugt, dass mit Galilei und Newton die Physik endlich den sicheren Gang einer Wissenschaft gefunden habe und von nun an die Wahrheit über die die Erscheinungswelt bevölkernden Entitäten und die herrschenden Naturgesetze enthüllen werde. ${ }^{350}$ Aber genau dieses „Urvertrauen" steht in der wissenschaftstheoretischen Realismusdebatte auf dem

\footnotetext{
${ }^{348}$ Kant ([1781/1787], 1998), A 225-226, B 273.

${ }^{349}$ Hierbei fällt auf, dass diese Begründung unabhängig von Kants Transzendentalem Idealismus ist. Zumindest an dieser Stelle scheint es also so, als bestünde für Kant kein unmittelbarer Zusammenhang zwischen seinem Transzendentalen Idealismus und seinem epistemischen Optimismus.

${ }^{350}$ Natürlich ist dies ein verzeihlicher Fehler. Zu Kants Lebzeiten hatten sich alle, auch die überraschendsten Vorhersagen der Newton'schen Physik bestätigt.
} 
Prüfstand. Kant kann nichts zu dieser Debatte beitragen, da er nicht mehr tut, als an jenes zu appellieren, und Massimi gelingt es, wie wir sahen, ebenfalls nicht, überzeugende Argumente für eine kantische Wissenschaftsphilosophie vorzustellen. Aus wissenschaftstheoretischer Perspektive spricht somit nichts für die von ihr vorgeschlagene kantische Phänomenkonzeption.

\subsection{Zusammenfassung}

Michela Massimi versucht für eine neue Theorieoption in der Realismusdebatte zu werben: einen moderaten, kantischen Realismus. Dieser Position zufolge sind wissenschaftliche Phänomene keine von uns unabhängigen Sachverhalte, sondern begrifflich verfasste Erscheinungen in einem kantischen Sinne. Insbesondere am Beispiel von Galileis Untersuchung des Fallgesetzes versucht Massimi aufzuzeigen, dass die Art und Weise, wie Wissenschaftler in der Praxis vorgehen, eine solche Phänomenauffassung nahe legt. Massimis These ist, dass in wissenschaftlich relevanten Beobachtungsurteilen bereits kausale Begrifflichkeiten über unbeobachtbare Ursachen a priori vorausgesetzt werden müssen, da ansonsten keine wissenschaftliche Erkenntnis möglich sei. Darüber hinaus versucht sie in einem zweiten Schritt zu zeigen, dass durch diese Aufgabe der metaphysischen These des Wissenschaftlichen Realismus der epistemische Optimismus gerettet werden kann.

Allerdings wurde bei genauerer Prüfung ihrer Argumente deutlich, dass es Massimi nicht gelingt, überzeugende wissenschaftstheoretische Gründe für ihre Position anzuführen. Weder kann sie begründen, warum die Praxis, kausale Hypothesen zu bilden, gegen die metaphysische These des Wissenschaftlichen Realismus spricht, noch kann sie zeigen, dass dem Kantianer plausible Argumente für den epistemischen Optimismus zur Verfügung stehen, die dem Wissenschaftlichen Realisten fehlen. Damit muss man konstatieren, dass eine kantische Phänomenkonzeption nicht dazu in der Lage ist, der Realismusdebatte die von Massimi in Aussicht gestellten neuen Impulse zu vermitteln. 
